\title{
Publisher's Note: Development of improved radiation drive environment for high foot implosions at the National Ignition Facility [Phys. Rev. Lett. 117, 225002 (2016)]
}

D. E. Hinkel, L. F. Berzak Hopkins, T. Ma, J. E. Ralph, F. Albert, L. R. Benedetti, P. M. Celliers, T. Döppner, C. S. Goyon, N. Izumi, L. C. Jarrott, S. F. Khan, J. L. Kline, A. L. Kritcher, G. A. Kyrala, S. R. Nagel, A. E. Pak, P. Patel, M. D. Rosen, J. R. Rygg, M. B. Schneider, D. P. Turnbull, C. B. Yeamans, D. A. Callahan, and O. A. Hurricane (Received 3 February 2017; published 22 February 2017)

DOI: 10.1103/PhysRevLett.118.089902

This paper was published online on 23 November 2016 with an incomplete reference. Reference [10] should read as "[10] O. Jones, N. Izumi, L. B. Hopkins, D. J. Strozzi, P. A. Amendt, G. N. Hall, D. D. Ho, S. F. Khan, N. B. Meezan, J. D. Moody, S. R. Nagel, J. E. Ralph, and R. P. J. Town, Bull. Am. Phys. Soc. 59 (15), 66 (2014); P. Amendt, D. D. Ho, and O. S Jones, Phys. Plasmas 22, 040703 (2015); L. F. Berzak Hopkins, S. Le Pape, L. Divol, N. B. Meezan, A. J. Mackinnon, D. D. Ho, O. S. Jones, S. Khan, J. L. Milovich, J. S. Ross, P. Amendt, D. Casey, P. M. Celliers, A. Pak, J. L. Peterson, J. Ralph, and J. R. Rygg, Phys. Plasmas 22, 056318 (2015); O. S. Jones, C. A. Thomas, P. A. Amendt, G. N. Hall, N. Izumi, M. A. Barrios Garcia, L. F. Berzak Hopkins, H. Chen, E. L. Dewald, D. E. Hinkel, A. L. Kritcher, M. M. Marinak, N. B. Meezan, J. L. Milovich, J. D. Moody, A. S. Moore, M. V. Patel, J.E. Ralph, S. P. Regan, M. D. Rosen, M. B. Schneider, S. M. Sepke, D. J. Strozzi, and D. P. Turnbull, J. Phys. Conf. Ser. 717, 012026 (2016).” The reference has been corrected as of 13 February 2017. The reference is incorrect in the printed version of the journal. 